\title{
Design and Construction of Integrated Small-Diameter Drift Tube and Thin-Gap Resistive Plate Chambers for the Phase-1 Upgrade of the ATLAS Muon Spectrometer
}

\author{
H. Kroha on behalf of the ATLAS collaboration ${ }^{\mathrm{a}, *}$ \\ ${ }^{a}$ Max-Planck-Institute for Physics, Munich, Germany
}

\begin{abstract}
In the long shutdown 2019-2020 for the Phase-1 upgrade of the Large Hadron Collider (LHC), 16 new integrated muon tracking and trigger chambers will be installed at the ends of the toroid magnet coils in the small azimuthal sectors of the inner barrel layer (BIS) of the ATLAS muon spectrometer in order to improve the trigger selectivity and fake trigger suppression in the transition region $1.0<|\eta|<1.3$ between the barrel part and the endcaps. The new muon detectors consist of a small-diameter muon drift tube (sMDT) precision tracking chamber with $15 \mathrm{~mm}$ tube diameter and a pair of thin-gap RPC chambers with $1 \mathrm{~mm}$ gas gap width. The new integrated chamber modules (labelled BIS 78) are currently under construction and will replace the present BIS 7 and 8 MDT tracking chambers with $30 \mathrm{~mm}$ diameter drift tubes. The project is the pilot phase for the complete replacement of the small barrel inner layer MDT chambers with the new integrated tracking and trigger detectors in the ATLAS Phase-2 upgrade in $2024-2026$ in order to increase the barrel first-level muon trigger coverage and efficiency at the high luminosities at HL-LHC. The sMDT chambers have been chosen to make room for the new trigger chambers and because of their eight times higher rate capability than MDT chambers. The new thin-gap RPC chambers have about 15 times lower avalanche charges and correspondingly increased lifetime and rate capability at HL-LHC and will be operated in coincidence with the endcap trigger chambers. They consist a of triplet of gas gaps which has to be also very thin supported by a light-weight support structure which is interleaevd with the sMDT chamber supports.
\end{abstract}

Keywords: Muon chambers, ATLAS detector, small-diameter MDT chambers, thin-gap RPC chambers PACS: 29.40.Cs, 29.40.Gx

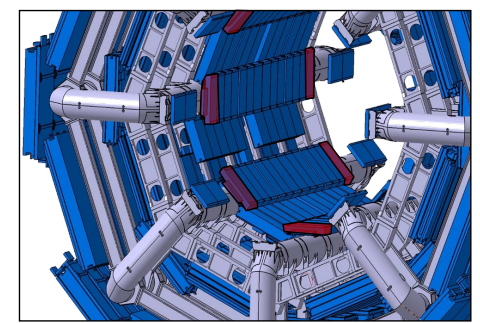

Figure 1: Location of the new BIS 78 muon tracking and triger chambers (red) in the small barrel inner layer of the ATLAS muon spectrometer.

\section{Chamber Layout and Design}

In the 2019-2020 shutdown of the LHC, 16 new integrated muon tracking and trigger chambers will be installed at the ends of the toroid magnet coils in the small sectors of the barrel inner layer (BIS) of the ATLAS muon spectrometer (see Figure 1). This upgrade will significantly improve the trigger selectivity and fake trigger suppression in the transition region $1.0<|\eta|<$ 1.3 between the barrel and endcaps (see Fig. 2).

The new BIS 78 chambers have to fit into a very confined space between the barrel toroid magnet of the ATLAS muon

\footnotetext{
${ }^{*}$ Corresponding author

Email address: kroha@mppmu.mpg.de (H. Kroha on behalf of the ATLAS collaboration)
}

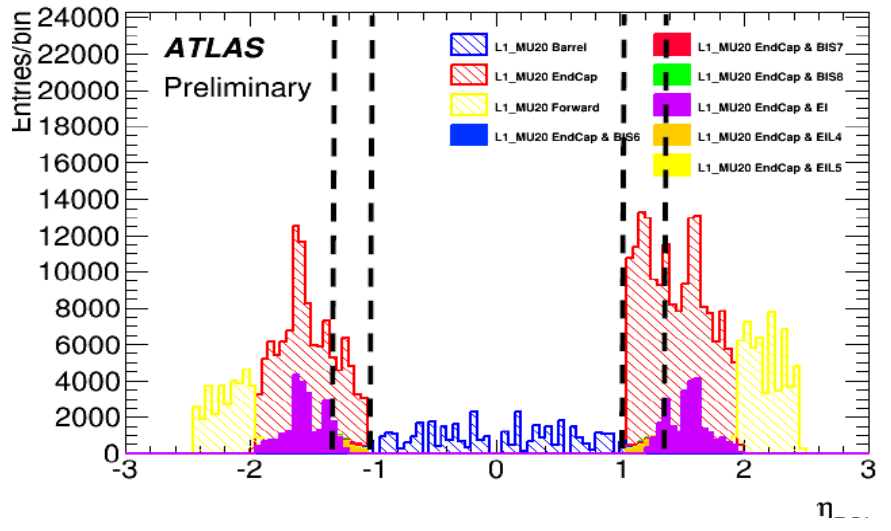

Figure 2: ATLAS Level-1 muon trigger rate distribution as function of $\eta$ from measurements in LHC Run-2 (hatched histograms). The accidental trigger rate in the transition region $1.0<|\eta|<1.3$ (dashed black lines) is strongly reduced to the level of the barrel trigger rate by including the new BIS 78 RPC trigger chambers together with the standard endcap TGC trigger chambers in the middle endcap layer. This combination contributes very small fake trigger rates indicated by the red and green full bars on top of the larger contributions in the yellow and purple full histograms from combination of the middle endcap layer with TGC chambers in the inner endcap layer EI. 


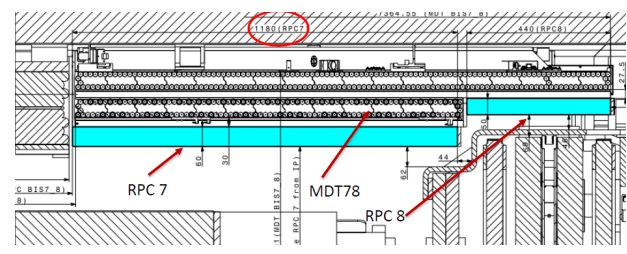

Figure 3: Integration of the new BIS 78 chambers in the overlap region between barrel and endcap muon spectrometer.

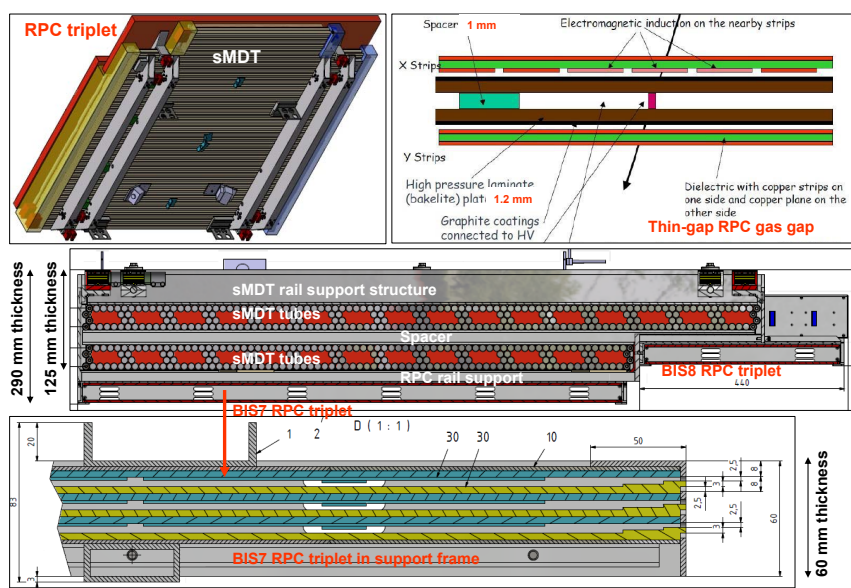

Figure 4: Design of the integrated BIS 78 sMDT and triple thin-gap RPC chambers.

spectrometer and the calorimeter and overlap with the inner wheels of the endcap muon spectrometer (see Figure 3). Therefore, the thickness of the chambers and also the lateral dimensions and the services and cables are tightly constrained. In order to make space for the new triplet RPC trigger chambers, which must not exceed $60 \mathrm{~mm}$ thickness for the BIS 7 and $50 \mathrm{~mm}$ for the BIS 8 part including the support frames, the present BIS 7 and 8 MDT chambers with $30 \mathrm{~mm}$ diameter drift tubes will be replaced by single small-diameter muon drift tube (sMDT) chambers [1] with $15 \mathrm{~mm}$ tube diameter which have been developed for this purpose. Another advantage of the sMDT chambers is their 8 times higher rate capability [1] compared to the MDT chambers which will not be fully exploited in ATLAS at HL-LHC. The integrated chambers (see Figure 4) have a height of $249 \mathrm{~mm}$ including the rail supports. Because of the overlap with the inner endcap wheels, the sMDT chambers have complex shapes with variations from sector to sector. These mechanically complex chambers can only be assembled with the required high sense wire positioning accuracy of better than $20 \mu \mathrm{m}$ by using the assembly method newly developed for the sMDT chambers [1]. The BIS 78 chambers contain either 648 or 744 drift tubes.

The new trigger detectors are thin-gap RPC chambers with $1 \mathrm{~mm}$ gas gap width compared to the $2 \mathrm{~mm}$ of the existing ATLAS RPC chambers and with high-pressure phenolic laminate (HPL) electrodes of only $1.2 \mathrm{~mm}$ thickness. With the smaller gas gap thickness and new high-sensitivity preamplifiers, the operating voltage can be reduced from 9.6 to $5.8 \mathrm{kV}$ and the avalanche charge decreased by a factor of about 15 which leads to an increase in lifetime by the same factor, allowing for more than 10 years of operation at HL-LHC using the same gas as the current RPCs. The rate capability reaches $10 \mathrm{kHz} / \mathrm{cm}^{2}$. The

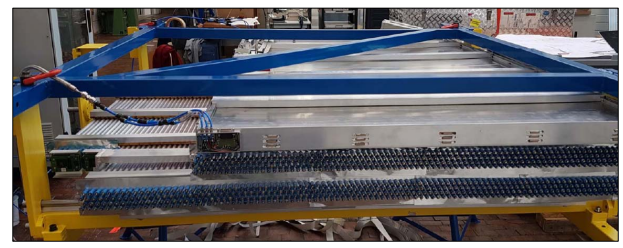

Figure 5: Assembly of a BIS 78 sMDT chamber (bottom) with a prototype triplet RPC chamber in its aluminum space frame (top) on their interleaved rail support structures.

highest background rates rates expected in the BIS layer at HLLHC are $600 \mathrm{~Hz} / \mathrm{cm}^{2}$. The BIS 78 trigger chambers consist of a triplet of gas gaps. Readout panels with othogonal strip electrodes for $\eta$ and $\phi$ coordinate measurement are attached to opposite sides of each gas gap. Each readout panel consists of a $0.3 \mathrm{~mm}$ thick PCB with copper strips pointing towards the gas gap and a PCB with continuous copper ground plane serving as Faraday cage separated by a $3 \mathrm{~mm}$ thick dielectric layer with refractive index $n \approx 1.2$. A gas gap with its two readout panels has a thickness of $11.7 \pm 0.7 \mathrm{~mm}$. Three of them are stacked inside an light-weight aluminum support frame of $60 \mathrm{~mm}$ height. The detector components are held together by $2 \mathrm{~mm}$ thick prebent aluminum sheets which keep the detector deformations within the $60 \mathrm{~mm}$ envelope and uniformly distribute forces. The total weight of the three RPC detectors is about $50 \mathrm{~kg}$.

\section{Chamber Construction and Test}

Four of the 16 BIS 78 sMDT chambers have already been assembled (see Figure 5). They carry optical sensors for monitoring of deformations and for global alignment in the barrel on the rail support side oriented towards the toroid coils and new optical alignment systems connecting them to the endcap wheels on the opposite surface pointing to the RPC. The sensor mounting platforms are glued to the outer tube layers on the assembly jig assembly with typically $100 \mu \mathrm{m}$ precision with respect to the sense wires. Afterwards, the platform positions are measured with $10 \mu \mathrm{m}$ precision with feeler gauges. Also the wire grid at both chamber ends is measured with feeler gauges on a flat granite table determining also gravitational sag and torsion around the tube axis. The mechanical wire position measurement makes use of precisely machined reference surfaces on the metal inserts of the injection molded plastic endplugs of the drift tubes which also hold the wires [1]. A three dimensional measurement of the endplugs of the first BIS 78 chamber has been performed with a large coordinate measuring machine based on the same principle. The measurement showed an overall wire positioning accuracy of $12 \mu \mathrm{m}$ (rms). The design of the RPC support frame is completed and the assembly of the RPC chambers will start in October 2018. A successful mechanical integration test of sMDT and RPC prototype chambers has been performed (see Figure 5).

\section{References}

[1] H. Kroha, et al., Construction and Test of New Precision Drift Tube Chambers for the ATLAS Muon Spectrometer, Nucl. Instr. and Meth. A 845 (2017) 244. 